\title{
ROSAT Temperatures and Abundances for a Complete Sample of Elliptical Galaxies
}

\author{
David S. Davis \& Raymond E. White III \\ Department of Physics \& Astronomy, University of Alabama, Tuscaloosa, AL 35487-0324
}

\begin{abstract}
We determine X-ray temperatures and abundances for elliptical galaxies drawn from a complete, optically selected sample. The optical magnitudelimited sample consists of 43 galaxies, complete to a (corrected) $B$ magnitude of $m_{B}^{o}=11.36$. Of these, 30 have enough X-ray spectral counts to allow temperature determinations. We find that the temperatures of the X-ray emitting gas in these ellipticals are correlated with the central stellar velocity dispersions: $T \propto \sigma^{1.45}$; this is a shallower trend than the simple thermal relation $T \propto \sigma^{2}$. The diffuse gas is substantially hotter than the kinetic temperature of the luminous stars, since $k T>\mu m_{p} \sigma^{2}$ for all galaxies with measurable temperatures. This strongly indicates that dark matter halos are characterized by velocity dispersions which exceed those of the luminous stars. We see no evidence of emission from X-ray binaries becoming progressively more dominant in lower luminosity ellipticals. We find that so-called "supersoft" sources adhere to the observed $k T-\sigma$ relation, so they are no softer than expected for their velocity dispersions. We also find that ISM temperatures and abundances are correlated, with the gas in hotter systems being more enriched than in cooler galaxies. However, no correlation is found between gaseous abundances and stellar abundances, as inferred from $M g_{2}$ indices.
\end{abstract}

Subject headings: galaxies: abundances - galaxies: elliptical — galaxies: ISM - X-ray: galaxies 


\section{Introduction}

Elliptical galaxies have been known as X-ray sources since several were detected in the Virgo cluster by the Einstein Observatory (Forman et al. 1979). Long and Van Speybroeck (1983) compiled many more Einstein detections of ellipticals and Nulsen, Stewart and Fabian (1984) showed that diffuse gas is their dominate source of X-ray emission. Analysis of Einstein Observatory IPC spectra shows that the emission from luminous ellipticals can be fit by thermal plasma models with fairly low temperatures, $0.5-2.0 \mathrm{keV}$ (Forman, Jones, \& Tucker 1985; Trinchieri, Fabbiano \& Canizares 1986). More recent Ginga, ROSAT and $A S C A$ observations of individual galaxies confirm these characteristic temperatures (cf. Awaki et al.1991; Forman et al. 1993; Fabbiano, Kim \& Trinchieri 1994; Awaki et al.1994; Matsushita et al.1994). The emission from lower luminosity ellipticals is apparently more complex, with evidence for both harder and "supersoft" emission according to Fabbiano, Kim \& Trinchieri (1992); these authors attribute the hard component to stellar X-ray binaries and suggest the supersoft emission may be from M stars, ultrasoft X-ray binaries and/or relatively cool ISM.

To date, there has been no study of the X-ray properties of a complete sample of normal elliptical galaxies. The IPC detector on the Einstein Observatory was used to observe $\sim 200$ normal galaxies, including many ellipticals (see the review by Fabbiano $1989)$, but these data allow only small $(N \approx 14)$ complete samples of ellipticals to be constructed. Furthermore, the limited energy resolution of the IPC prevented accurate temperature determinations. We have therefore turned to the longer exposures of $R O S A T$ pointed observations to construct the largest possible complete sample of accurate X-ray luminosities and temperatures for elliptical galaxies. In this letter we present new gas temperature and abundance determinations from ROSAT PSPC spectra of a subset of this complete sample and explore their relation to optical and dynamical properties of the host galaxies. We will present detailed analyses of their X-ray luminosities in a forthcoming paper (Davis \& White 1996).

\section{Sample Selection}

We used the HEASARC BROWSE software to select our optical magnitude-limited sample from the Third Revised Catalog of Galaxies (de Vaucouleurs et al. 1991; hereafter $R C 3$ ), using its corrected $B$ magnitudes. We limited our sample to elliptical galaxies (de Vaucouleurs T-types $-4,-5 \&-6)$. We then used BROWSE to cross-correlate this sample with the ROSAT and Einstein pointed observations. Neglecting galaxies in the zone-of avoidance (taken here to be $|b|<20^{\circ}$ ), we found that 38 of the 43 optically brightest 
ellipticals have ROSAT PSPC data, and 4 of the remaining 5 have Einstein IPC data (the remaining galaxy, NGC 7507, will soon have ROSAT HRI data available, but this has no spectral information); since ROSAT PSPC data have better spectral resolution than Einstein IPC data, they are preferable when there is a choice. The sample of 43 optically-selected ellipticals is complete to a limiting (corrected) blue magnitude of $m_{B}^{0}=11.36$ and includes four Local Group dwarfs. The X-ray counterpart to our optical sample is thus $98 \%$ complete, with X-ray data for 42 of the 43 ellipticals. If we press to fainter optical magnitudes, the marginal completeness in the X-ray data deteriorates at the $50 \%$ level for the next 10 or so optically brightest galaxies. In this letter we present a subset of 30 galaxies from this complete sample which have sufficient spectral counts to accurately determine their X-ray temperatures; 27 of these galaxies also have well-constrained abundances.

\section{X-Ray Data}

In order to tie the X-ray properties of ellipticals to their optical properties in a uniform manner, we let the optical photometry dictate the spatial size of the X-ray spectral extraction regions. For each galaxy in our optically selected sample, we extract X-ray spectra from within $6 r_{e}$, where $r_{e}$ is the galaxy's optical effective radius (derived from the $R C 3)$. Due to X-ray scattering in the ROSAT mirror assembly, the minimum suggested extraction radius for point sources is $1.5^{\prime}$, which is the $99.5 \%$ encircled energy radius for a 1 $\mathrm{keV}$ spectrum. Our minimum metric extraction radius must be $3 r_{e}$ to meet this requirement with the current sample. The purpose of our larger metric extraction radius of $6 r_{e}$ is to provide more global X-ray properties, for better comparison with global optical quantities such as magnitudes $\left(6 r_{e}\right.$ encloses $91 \%$ of a de Vaucouleurs $r^{1 / 4}$ profile).

For the PSPC data, source spectra were selected from observation times when the Master Veto Rate $(M V R)$ was within recommended bounds: $20<M V R<170$. The background for each spectrum was obtained from an annulus around the galaxy extending from $6 r_{e}$ to $12 r_{e}$ from its center. All detectable point sources were excluded from both source and background spectra; excluded points in the source regions tended to be in the outskirts so they are unlikely to be X-ray binaries. Source and background spectra were also corrected for vignetting effects and for the residual particle background (in the manner prescribed by Snowden et al. 1994), which is proportional to the $M V R$. To ensure correct Poisson errors were used throughout, we modified the appropriate data header as outlined in the PROS QPSPEC documentation. Finally, the extracted spectra were rebinned so that each channel has a minimum of 25 counts, and systematic errors of $1 \%$ were included. 


\section{Analysis}

We used XSPEC 9.0 software (Schafer et al. 1990) to fit various models to the extracted spectra. An isothermal Raymond-Smith plasma model was our benchmark model, with the temperature and abundance allowed to vary in the fits. We also included a variable absorption component due to the column density of Galactic hydrogen in the line-of-sight. The redshift of the model spectrum was fixed to that corresponding to the heliocentric velocity of the galaxy given in the $R C 3$. The extracted spectra were fit between $\sim 0.2$ and $2.0 \mathrm{keV}$, the exact energy boundaries being set by the channel grouping. Once a minimum in $\chi^{2}$ was found, the $90 \%$ confidence errors were determined for the free parameters. Most of the galaxies have sufficient counts so that the temperature, abundance and absorption could all be fit. For a few galaxies, however, the number of free parameters had to be reduced to allow reasonable errors to be determined. For such galaxies we first fixed the abundance to 0.2 solar; when necessary, we also fixed the absorption to the Galactic value (Stark et al. 1992). The Raymond-Smith thermal model provided formally excellent fits to all but two galaxies, with $\chi^{2}$ per degree of freedom $\nu$ of $\chi_{\nu}^{2} \approx 1$. The two exceptions (NGC 4486 \& IC 1459) are known to have AGN, so we fit composite models consisting of a Raymond-Smith isothermal plus a power-law spectral component; these composite spectra achieved $\chi_{\nu}^{2} \approx 1$. The detailed spectral fitting results are shown in Table 1 , where the indicated errors are $90 \%$ confidence limits.

\section{1. $\quad k T-\sigma$ Correlation}

For the 30 galaxies which have enough counts to allow accurate temperature determinations, Fig. 1 shows a logarithmic plot of their temperatures $k T$ against $\sigma$, the central line-of-sight stellar velocity dispersions within the galaxies (from Faber et al. 1989 or Dressler, Faber \& Burstein 1991). Characteristics of the best fit linear regression to these data are shown in the first line of Table 2. We found that three galaxies (M32, NGC 1052 and NGC 2768) have temperatures of $\sim 3-4 \mathrm{keV}$, which are much greater than those of other galaxies with similar velocity dispersions. These galaxies are likely to have their X-ray emission dominated by X-ray binaries or AGN (cf. Eskridge, White \& Davis 1996; Ho, Filipenko \& Sargent 1995), rather than by the cooler diffuse gas expected from the accumulation of stellar ejecta. Neglecting these three galaxies, the best fit linear regression yields $k T \propto \sigma^{1.45 \pm 0.20}$ and the temperature dispersion is only 0.10 dex (see line 2 of Table 2); the regression is shown as a solid line in Figure 1. This correlation is shallower than what would obtain if the gas and stars had the same kinetic temperature: $k T=\mu m_{p} \sigma^{2}$; this latter relation is also shown in Fig. 1 as a dashed line. The bulk of our 
temperature measurements indicate that warm diffuse gas is being detected, even down to low luminosities and velocity dispersions where some investigators expect such gas to be swept by winds (e.g. David, Forman \& Jones 1991; Ciotti et al. 1991). Fig. 1 also shows that the global gas temperatures are hotter than the kinetic temperatures of luminous stars in the galaxies, since $k T>\mu m_{p} \sigma^{2}$ in all the galaxies.

\subsection{Temperature-Abundance Correlation}

For those 27 galaxies with enough counts for elemental abundances to be constrained, we find that the measured abundances (driven by $\sim 1 \mathrm{keV}$ Fe L emission in $R O S A T$ PSPC spectra) are correlated with the gas temperatures: hotter gas tends to have higher abundances (see Fig. 2). Line 3 of Table 2 shows the regression results for all the data. When we exclude M32 and NGC 1052 from the regression (for being hot point sources, rather than diffuse gas), we find the abundance $A \propto T^{2.44 \pm 0.69}$ (see line 4 of Table 2), with a dispersion of $0.48 \mathrm{dex}$ in $A$. This latter correlation is shown as a dotted line in Fig. 2 (where M32 and NGC 1052 are also indicated). We have checked our abundances against the several published $A S C A$ values and find that in most cases they agree within the $90 \%$ confidence level and in all cases they agree within the $99 \%$ confidence level. Our PSPC temperatures are also consistent with the $A S C A$ results. We find no correlation between our measured abundances and the stellar abundances of the host galaxies, as inferred from $M g_{2}$ indices (7S) (see lines 5-6 of Table 2); this is consistent with the $A S C A$ results of Loewenstein et al. (1994) and Mushotzky et al. (1994).

\section{Summary and Discussion}

In this letter we have shown that gas temperatures are correlated with stellar velocity dispersions, $T \propto \sigma^{1.45}$, and are everywhere hotter than the stellar kinetic temperatures $\left(k T>\mu m_{p} \sigma^{2}\right)$. There are several possible mechanisms for the gas acquiring more specific energy than the stars. One possibility is that supernovae have heated the gas, but this is inconsistent with the generally low metal abundances being found in such galaxies (Matsushita et al. 1994; Loewenstein et al. 1994; and $\S 4.2$ of this paper). A more likely possibility is that the luminous parts of the galaxies are embedded in dark matter halos which are dynamically hotter than the stars (Fabian et al. 1986). As stellar ejecta accumulates and flows inward in cooling flows, movement through the gravitational potential heats the gas. If dark matter halos are characterized by velocity dispersions that are $\gtrsim 50 \%$ greater than those of the luminous stars, then gravitational heating can raise the 
gas temperatures to the observed values.

In the largest study of X-ray spectral properties of galaxies to date, Kim, Fabbiano \& Trinchieri (1992, hereafter KFT) found that the X-ray emission from elliptical galaxies is more complicated than the simple thermal plasma found by Forman et al. (1985). KFT found for galaxies with high X-ray to optical flux ratios $\left(L_{X} / L_{B}\right)$ that their spectra are indeed well described by Raymond-Smith thermal plasma models with temperatures of $\sim 1 \mathrm{keV}$ and solar abundances. But for galaxies with intermediate values of $L_{X} / L_{B}, \mathrm{KFT}$ found spectral hardening. At even lower values of $L_{X} / L_{B}$, the spectra require a a very cool "supersoft" $0.2 \mathrm{keV}$ component in addition to the hard $(\sim 5 \mathrm{keV})$ component. Although a few galaxies in our sample have unusually hard emission, we find that the correlation of temperature with velocity dispersion persists down to $k T \approx 0.2 \mathrm{keV}, L_{B} \approx 4.7 \times 10^{9} h^{-2}$ $L_{\odot}, \sigma \approx 120 \mathrm{~km} \mathrm{~s}^{-1}$. Our sample includes some of the galaxies deemed to have "supersoft" spectra by KFT, but we find that these sources are simply on the cool end of a continuous distribution, with temperatures consistent with expectations from ISM in galaxies with low velocity dispersions (hence, shallower gravitational potentials).

While a few of the galaxies in our sample exhibit hard emission (due to X-ray binaries, AGN, or both) we find no general hardening of the X-ray emission as we go to lower luminosities and velocity dispersions, as might be expected if low-luminosity ellipticals are blowing winds. KFT find harder spectra for a subset of their sample (their "Group 2"), which they attribute to X-ray binaries, but our PSPC data for 4 of 8 galaxies in this subsample (after removing NGC 1052, a Seyfert 2, which may have biased the results of KFT) show no evidence for having harder spectra attributable to X-ray binaries. Thus, we detect gaseous $\mathrm{X}$-ray emission without contamination by $\mathrm{X}$-ray binaries down to $L_{B} \approx 4.7 \times 10^{9} h^{-2} L_{\odot}, L_{X} \approx 10^{39} h^{-2} \mathrm{erg} \mathrm{s}^{-1}$ and $\sigma \approx 120 \mathrm{~km} \mathrm{~s}^{-1}$. This is contrary to the predictions of some theoretical models for the evolution of hot gas in ellipticals, in which the threshold for the onset of supernovae-driven winds occurs at significantly higher optical luminosities (Ciotti et al. 1991; David et al. 1991). Such winds drive out the gas, leaving only X-ray binaries as significant sources of X-ray emission. M32 may be an example of such a wind-blowing galaxy, since it exhibits hard X-ray emission and is ten times less luminous (both optically and in X-rays) than the next less luminous galaxy in our sample.

Finally, we find that abundances are correlated with global temperatures: $A \propto T^{2.4}$. This may reflect an underlying trend in observed stellar abundances (more luminous galaxies are more metal rich), but we find no detailed correlation between our gaseous abundances and the stellar metallicity index $M g_{2}$. The $M g_{2}$ index is measured within the inner few kpc of galaxies, so it may not be representative of the galaxy out to our extraction radius of $6 r_{e}$. For example, Davies, Sadler \& Peletier (1993) find that the $M g_{2}$ index drops by $\sim 25 \%$ 
from the center to a radius of $\sim 1 r_{e}$. Analysis of $A S C A$ observations of the elliptical NGC 4636 shows there is a gaseous abundance gradient evident from spatially resolved X-ray spectroscopy, a gradient which persists to at least $5 r_{e}$ (Mushotzky et al. 1994). Our global, emission-weighted measures of abundances obscure such structure. The lack of correlation between the stellar and gaseous abundances may alternatively indicate that more luminous galaxies have larger supernova rates than less luminous galaxies, but not so large as to unbind the gas.

In a subsequent, more detailed analysis of this complete sample of ellipticals, we will present their X-ray luminosities, their luminosity function, discuss the Local Group dwarf galaxies, and assess additional correlations and implications.

This research made use of the HEASARC, NED, and SkyView databases. We would like to thank Keith Arnaud for his help with the IPC data. This work was partially supported by the NSF and the State of Alabama through EPSCoR grant EHR-9108761. REW also acknowledges partial support from NASA grants NAG 5-1718 and NAG 5-1973.

\section{REFERENCES}

Awaki, H., Koyama, K., Kuneida, H., Takano, S. \& Tawara, Y. 1991, ApJ, 366, 88

Awaki, H., et al. 1994, PASJ, 46, L65

Ciotti, L., Pellegrini, S., Renzini, A., \& D‘Ercole, A. 1991 ApJ, 376, 380

David, L. P., Forman, W. \& Jones, C. 1991, ApJ, 369, 121

Davies, R. L., Sadler, E. M. \& Peletier, R. F. 1993, M.N.R.A.S, 262, 650

Davis, D.S. \& White, R.E. III 1996, in preparation (Paper II)

de Vaucouleurs, G., de Vaucouleurs, A., Corwin, H. G., et al., Third Reference Catalogue of Bright Galaxies, Springer 1991

Dressler, A., Faber, S. M. \& Burstein, D. 1991, ApJ, 368, 54 (7S)

Eskridge, P.B., White, R.E. III \& Davis, D.S. 1996, ApJ, 463, L59

Fabbiano, G., Kim, D.-W., \& Trinchieri, G. 1992, ApJS, 80, 531

Fabbiano, G., Kim, D.-W., \& Trinchieri, G. 1994, ApJ, 429, 94

Fabbiano, G. 1989, ARAA, 27, 87

Faber, S. M., Wegner, G., Burstein, D., Davies, R. L., Dressler, A., Lynden-Bell, D. \& Terlevich, R. J. 1989, ApJS, 69, 763 (7S) 
Fabian, A.C., Thomas, P.A., Fall, S.M., \& White, R.E. III 1986, MNRAS, 221, 1049

Forman, W., Jones, C., David, L., Franx, M., Makishima, K., \& Ohashi, T. 1993, ApJ, L55

Forman, W., Schwarz, J., Jones, C., Liller, W. \& Fabian, A. C. 1979, ApJ, 234, L27

Forman, W., Jones, C. \& Tucker, W. 1985, ApJ, 293, 102

Forman, W., Jones, C. \& Tucker, W. 1994, ApJ, 429, 77

Ho, Filipenko \& Sargent 1995, ApJS, 98, 477

Kim, D.-W., Fabbiano, G. \& Trinchieri, G. 1992, ApJ, 393, 134

Loewenstein M., et al. 1994, ApJ, 436, L75

Long, K.S. \& Van Speybroeck, L.P. 1983, in Accretion-Driven X-ray Sources, eds. W. Lewin, E.P.J. van den Heuvel (Cambridge: Cambridge University Press), p.117

Matsushita, K. et al. 1994, ApJ, 436, L41

Mushotzky et al. 1994, ApJ, 436, L79

Nulsen, P. E., Stewart, G. C. \& Fabian, A. C. 1984, MNRAS, 208, 185

Schafer, R. A., Halberl, F., Arnaud, K. A. \& Tennant, A. F. 1990, XSPEC User Guide

Snowden, S. L., McCammmon, D., Burrows, D. N., \& Mendenhall, J. A. 1994, ApJ, 424, 714

Stark, A. A., Gammie, C. F., Wilson, R. W., Bally, J., Linke, R. A., Heiles, C. \& Hurwitz, M. 1992, Ap. J. Supp., 79, 77

Trinchieri, G., Fabbiano, G. \& Canizares, C. R. 1986, ApJ, 310, 637 
Table 1. Spectral Fits ${ }^{\mathrm{a}}$

\begin{tabular}{|c|c|c|c|c|c|}
\hline Name & $\begin{array}{c}k T \\
(\mathrm{keV})\end{array}$ & $\begin{array}{c}\text { Abundance } \\
\text { (solar) }\end{array}$ & $\begin{array}{l}N_{H}^{\mathrm{b}} \\
\text { fitted }\end{array}$ & $\begin{array}{c}N_{H}^{\mathrm{b}} \\
\text { Galactic }\end{array}$ & $\chi^{2} / \nu$ \\
\hline N 221 & $3.94_{-1.06}^{+1.73}$ & $0.44_{-0.31}^{+0.72}$ & $7.18_{-0.87}^{+1.16}$ & 6.50 & $90 / 104$ \\
\hline N 720 & $0.58_{-0.06}^{+0.05}$ & $0.10_{-0.04}^{+0.06}$ & $2.16_{-0.72}^{+0.73}$ & 1.42 & $64 / 56$ \\
\hline N 1052 & $2.88_{-1.25}^{+5.92}$ & $<1.09$ & $3.23_{-1.60}^{+5.57}$ & 2.90 & $14 / 26$ \\
\hline N 1399 & $1.08_{-0.01}^{+0.02}$ & $1.04_{-0.20}^{+0.26}$ & $1.03_{-0.24}^{+0.25}$ & 1.39 & $152 / 155$ \\
\hline N 1395 & $0.82_{-0.06}^{+0.04}$ & $0.19_{-0.09}^{+0.13}$ & $1.55_{-0.65}^{+0.85}$ & 1.74 & $50 / 49$ \\
\hline N 1404 & $0.62_{-0.02}^{+0.03}$ & $0.22_{-0.05}^{+0.06}$ & $1.69_{-0.35}^{+0.34}$ & 1.39 & $103 / 114$ \\
\hline N 1407 & $0.85_{-0.15}^{+0.07}$ & $0.10_{-0.06}^{+0.07}$ & $9.35_{-5.30}^{+7.73}$ & 5.17 & $66 / 75$ \\
\hline N 1549 & $0.48_{-0.16}^{+0.26}$ & $0.03_{-0.03}^{+0.11}$ & $0.72_{-0.72}^{+1.40}$ & 1.93 & $10 / 12$ \\
\hline N 2768 & $2.87_{-2.37}^{+16.13}$ & 0.20 & 1.87 & 4.26 & $1.5 / 5$ \\
\hline N 3557 & $0.79_{-0.39}^{+0.12}$ & $<2.77$ & 8.04 & 8.04 & $5.0 / 5$ \\
\hline N 3585 & $0.40_{-0.13}^{+0.46}$ & $0.11_{-0.11}^{+4.89}$ & 5.38 & 5.38 & $0.6 / 2$ \\
\hline N 3923 & $0.47_{-0.19}^{+0.16}$ & $0.09_{-0.05}^{+0.11}$ & $17.90_{-2.77}^{+28.38}$ & 6.37 & $37 / 47$ \\
\hline $\mathrm{N} 4105^{\mathrm{c}}$ & $0.76_{-0.69}^{+4.40}$ & 0.2 & 6.10 & 6.10 & $3.9 / 5$ \\
\hline N 4125 & $0.42_{-0.09}^{+0.14}$ & $0.06_{-0.03}^{+0.05}$ & 1.90 & 1.90 & $29 / 27$ \\
\hline N 4261 & $0.83_{-0.06}^{+0.05}$ & $0.09_{-0.04}^{+0.06}$ & $2.56_{-0.61}^{+0.71}$ & 1.88 & $54 / 61$ \\
\hline N 4278 & $0.64_{-0.27}^{+0.00}$ & $0.02_{-0.02}^{+0.04}$ & $2.65_{-2.50}^{+4.20}$ & 1.96 & $0.7 / 3$ \\
\hline N 4365 & $0.88_{-0.29}^{+0.60}$ & $0.01_{-0.01}^{+0.13}$ & $1.73_{-1.11}^{+1.38}$ & 1.60 & $14 / 21$ \\
\hline N 4374 & $0.74 \pm 0.05$ & $0.18_{-0.06}^{+0.16}$ & $2.72_{-1.02}^{+0.69}$ & 1.74 & $90 / 92$ \\
\hline N 4406 & $0.89 \pm 0.01$ & $0.37 \pm 0.05$ & $2.21_{-0.23}^{+0.24}$ & 2.61 & $161 / 167$ \\
\hline N 4472 & $1.01 \pm 0.02$ & $1.12_{-0.24}^{+0.35}$ & $1.17_{-0.32}^{+0.34}$ & 1.64 & $166 / 160$ \\
\hline $\mathrm{N} 4486^{\mathrm{d}}$ & $1.32_{-0.04}^{+0.03}$ & $0.41 \pm 0.05$ & $1.50_{-0.52}^{+0.65}$ & 1.70 & $16 / 16$ \\
\hline N 4494 & $0.20_{-0.04}^{+0.08}$ & 0.20 & 1.54 & 1.54 & $1.0 / 3$ \\
\hline N 4552 & $0.74_{-0.06}^{+0.07}$ & $0.08_{-0.03}^{+0.07}$ & $3.10_{-0.80}^{+0.77}$ & 2.50 & $55 / 51$ \\
\hline N 4636 & $0.72_{-0.02}^{+0.03}$ & $0.33_{-0.08}^{+0.15}$ & $\begin{array}{l}1.90_{-0.53}^{+0.49} \\
\end{array}$ & 1.87 & $118 / 131$ \\
\hline N 4649 & $0.86_{-0.01}^{+0.02}$ & $0.52_{-0.17}^{+0.32}$ & $2.03_{-0.75}^{+0.78}$ & 2.40 & $107 / 101$ \\
\hline N 4697 & $0.49_{-0.08}^{+0.12}$ & $<0.01$ & $2.78_{-0.77}^{+0.76}$ & 2.54 & $62 / 65$ \\
\hline N 4696 & $1.32_{-0.05}^{+0.04}$ & $0.86_{-0.22}^{+0.30}$ & $10.15_{-1.24}^{+1.70}$ & 8.79 & $210 / 177$ \\
\hline N 5322 & $0.51_{-0.14}^{+0.18}$ & $0.04_{-0.03}^{+0.08}$ & $<2.34$ & 1.87 & $20 / 21$ \\
\hline N 5846 & $0.76_{-0.04}^{+0.03}$ & $0.29_{-0.09}^{+0.14}$ & $4.89_{-1.15}^{+1.62}$ & 4.23 & $157 / 148$ \\
\hline I $1459^{\mathrm{d}}$ & $0.60_{-0.13}^{+0.12}$ & $0.31_{-0.15}^{+0.66}$ & 1.40 & 1.40 & $13 / 24$ \\
\hline
\end{tabular}

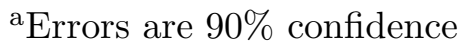

${ }^{\mathrm{b}} N_{H}$ in units of $10^{20} \mathrm{~cm}^{-2}$

${ }^{\mathrm{c}}$ Einstein IPC data used

${ }^{\mathrm{d}}$ A power law component was fit along with the Raymond-Smith plasma model 
Table 2. Correlations

\begin{tabular}{lllccccc}
\hline \hline & $\begin{array}{c}\text { Dependent } \\
\text { Variable }\end{array}$ & $\begin{array}{c}\text { Independent } \\
\text { Variable }\end{array}$ & Slope & $\begin{array}{c}\text { Dispersion } \\
\text { (Dep. Var.) }\end{array}$ & $\mathrm{N}$ & Prob $^{\mathrm{a}}$ & $\begin{array}{c}\text { Galaxies } \\
\text { Excluded }\end{array}$ \\
\hline 1) & $\log k T$ & $\log \sigma$ & $-0.20 \pm 0.36$ & 0.26 & 30 & 0.034 & \\
2) & $\log k T$ & $\log \sigma$ & $1.45 \pm 0.20$ & 0.10 & 27 & $9.5^{-5}$ & $\mathrm{M} 32, \mathrm{~N} 1052, \mathrm{~N} 2768$ \\
& & & & & & & \\
3) & $\log A$ & $\log k T$ & $1.34 \pm 0.46$ & 0.50 & 26 & $1.7^{-3}$ & \\
4) & $\log A$ & $\log k T$ & $2.44 \pm 0.69$ & 0.48 & 24 & $3.1^{-3}$ & $\mathrm{M} 32, \mathrm{~N} 1052$ \\
& & & & & & & \\
5) & $\log A$ & $M g_{2}$ & $-2.00 \pm 3.81$ & 0.58 & 26 & 0.85 & \\
$6)$ & $\log A$ & $M g_{2}$ & $2.18 \pm 6.68$ & 0.59 & 25 & 0.84 & $\mathrm{M} 32$ \\
\hline
\end{tabular}

aProbability that there is no correlation, using Kendall's $\tau$ test; dexponents are given as exponents.

\section{Figure Captions}

Fig. 1.- The $k T-\sigma$ correlation. The solid line is the fit to the data excluding the three galaxies with $k T>2 \mathrm{keV}$ (M32, NGC 1052, and NGC 2768). The dashed line shows the relation expected if the gas temperature and stellar kinetic temperatures were the same. The datum with the largest error bars (at $\log \sigma \approx 2.42$ ) is NGC 4105, the only galaxy in the plot where the temperature was determined using IPC data.

Fig. 2.- The abundance-temperature correlation. The dashed line is the fit to the sample excluding the high temperature galaxies M32 and NGC 1052 (seen at right). Kendall's $\tau$ indicates the correlation is significant at the $99.84 \%$ confidence level. 


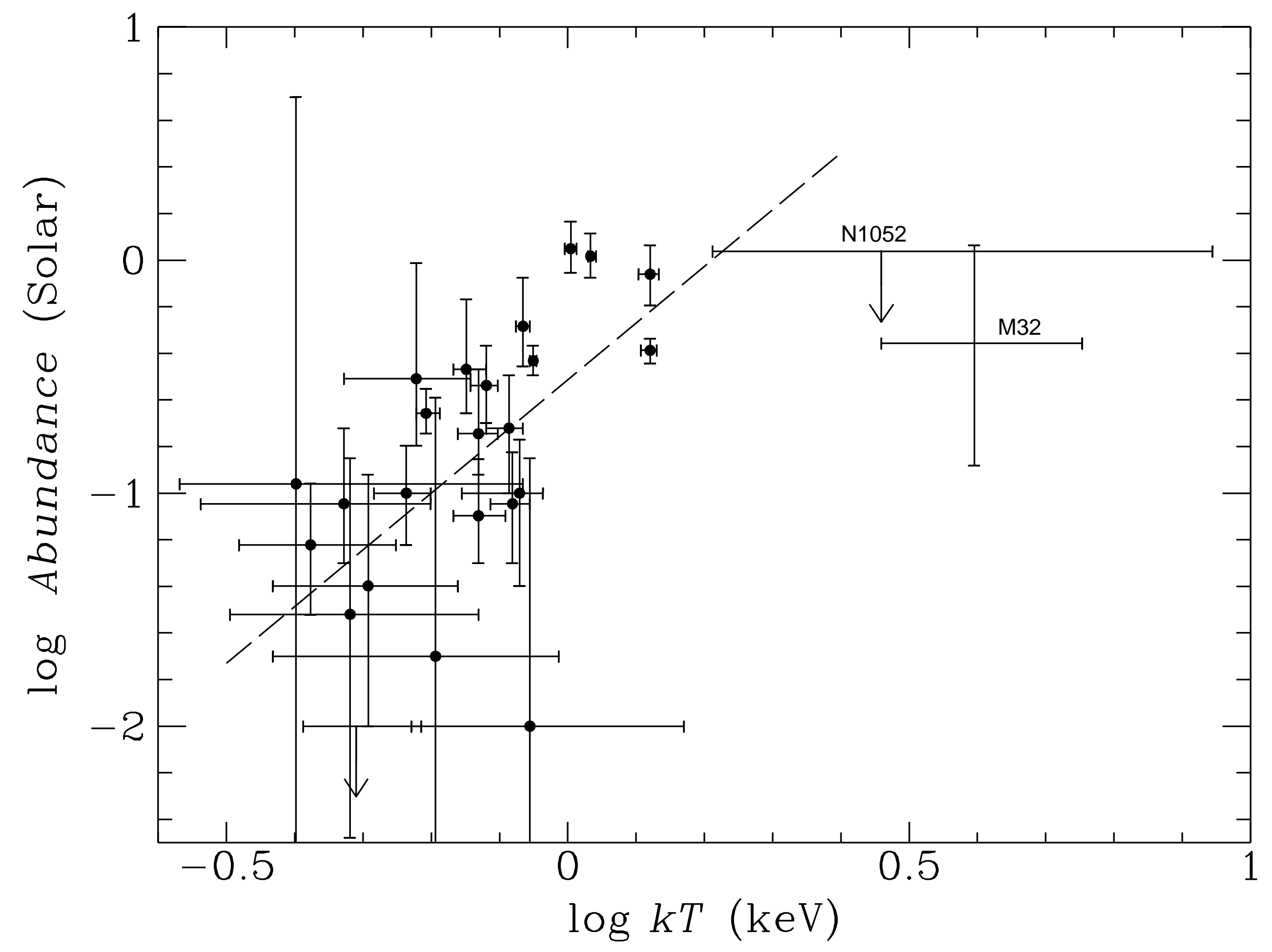

